\title{
Article \\ Effects of Community-Based Physical-Cognitive Training, Health Education, and Reablement among Rural Community-Dwelling Older Adults with Mobility Deficits
}

\author{
Chen-Yi Song $1, *\left(\mathbb{D}\right.$, Pay-Shin Lin ${ }^{2,3,4}$, Pei-Lun Hung ${ }^{1}$ and ADLers Occupational Therapy Clinic ${ }^{5}$ \\ 1 Department of Long-Term Care, National Taipei University of Nursing and Health Sciences, \\ Taipei 112303, Taiwan; ax911142@gmail.com \\ 2 Department of Physical Therapy, Graduate Institute of Rehabilitation Science, College of Medicine, \\ Chang Gung University, Taoyuan 33302, Taiwan; pslin@mail.cgu.edu.tw \\ 3 Master Degree Program in Healthcare Industry, College of Medicine, Chang Gung University, \\ Taoyuan 33302, Taiwan \\ 4 Healthy Aging Research Center, Chang Gung University, Chang Gung Memorial Hospital, \\ Taoyuan 33302, Taiwan \\ 5 ADLers Occupational Therapy Clinic, Taipei 10491, Taiwan; adlers0615@gmail.com \\ * Correspondence: cysong@ntunhs.edu.tw
}

\section{check for}

updates

Citation: Song, C.-Y.; Lin, P.-S.; Hung, P.-L.; ADLers Occupational Therapy Clinic. Effects of Community-Based PhysicalCognitive Training, Health Education, and Reablement among Rural Community-Dwelling Older Adults with Mobility Deficits. Int. J. Environ. Res. Public Health 2021, 18, 9374. https://doi.org/10.3390/ijerph18179374

Academic Editor: Juh Hyun Shin

Received: 22 June 2021

Accepted: 31 August 2021

Published: 5 September 2021

Publisher's Note: MDPI stays neutral with regard to jurisdictional claims in published maps and institutional affiliations.

Copyright: (c) 2021 by the authors. Licensee MDPI, Basel, Switzerland. This article is an open access article distributed under the terms and conditions of the Creative Commons Attribution (CC BY) license (https:// creativecommons.org/licenses/by/ $4.0 /)$.

\begin{abstract}
Reablement services are approaches for maintaining and improving the functional independence of older adults. Previous reablement studies were conducted in a home environment. Due to the limited evidence on the effects of multicomponent interventions and reablement in a community-based context, this study aimed to develop and evaluate the effect of community-based physical-cognitive training, health education, and reablement (PCHER) among rural communitydwelling older adults with mobility deficits. The trial was conducted in rural areas of New Taipei City, Taiwan. Older adults with mild to moderate mobility deficits were recruited from six adult daycare centers, and a cluster assignment was applied in a counterbalanced order. The experimental group $(n=16)$ received a PCHER intervention, comprising $1.5 \mathrm{~h}$ of group courses and $1 \mathrm{~h}$ of individualized reablement training, while the control group $(n=12)$ underwent PCHE intervention, comprising $1.5 \mathrm{~h}$ of group courses and $1 \mathrm{~h}$ of placebo treatment. A 2.5-h training session was completed weekly for 10 weeks. The outcome measures contained the de Morton Mobility Index (DEMMI), the Saint Louis University Mental Status (SLUMS) Examination, the Barthel Index (BI), the Short Physical Performance Battery (SPPB), and the Canadian Occupational Performance Measure (COPM). The PCHER significantly improved the DEMMI, SLUMS, BI, SPPB, and COPM (all $p<0.05$ ), with medium-to-large effect sizes. PCHER also showed an advantage over PCHE in terms of the SPPB $(p=0.02)$. This study verified that combining individualized reablement with group-based multicomponent training was superior to group courses alone in enhancing the functional abilities of community-dwelling older adults with mobility deficits.
\end{abstract}

Keywords: restorative care; reablement; function; elderly; long-term care

\section{Introduction}

Reablement services are approaches for maintaining and improving the functional independence of older adults [1]. The intervention is targeted, focused on enhancing the performance of daily activities defined as important by the person, and takes place in the person's home and local surroundings [2]. The aim is to enable people to age in place, be active and participate socially and in society [2]. Previous studies demonstrated some positive impacts of reablement on functional abilities, health-related quality of life, and reduction in healthcare service utilization [3-5]. For older adults with mild disability, reablement was found to enhance independence from long-term care services [6]. However, 
most reablement studies were conducted in the context of home care [1-5]. Communitydwelling older people with physical dysfunction may use other care services, such as adult day care. There is limited knowledge regarding reablement in a community-based context.

A one-year follow-up study reported that limitations in lower limb function is a risk factor for functional decline among older adults in rural areas [7]. As lower extremity function may predict future disability [8], improving functional mobility deficits to prevent further disability progression is vital. Physical exercise has been proposed for improving physical performance in the community-dwelling elderly, including those in daycare centers [9-11]. On the other hand, rural areas in Taiwan have relatively low financial resources and less access to multiple services. Older adults in rural communities have various health problems, including sarcopenia, cognitive impairment, malnourishment, and a susceptibility to falls [12-15].

Multicomponent interventions are generally more effective than single-component interventions. Combined physical and cognitive training, either simultaneous or subsequent, is more successful relative to physical or cognitive exercises alone [16]. Reablement programs combined with standard care (e.g., long-term care services such as home help or day care services) were found to be superior to standard care alone in enhancing the independence of older adults [6]. In Cho et al. [17], a multicomponent intervention program, comprising an exercise component (focusing on balance and muscle strength) and a fall education component, reduced the fall risk for community-dwelling older adults. The aforementioned studies implied that combining physical-cognitive training, health education, and reablement (PCHER) is beneficial to rural community-dwelling older adults.

The purpose of the present study was to develop and evaluate the effect of a communitybased intervention combining PCHER among rural community-dwelling older adults with mobility deficits compared with a control in relation to mobility, cognitive function, activities of daily living (ADL) function, physical function, and self-perceived activity performance and satisfaction with performance.

\section{Materials and Methods}

\subsection{Study Design and Participants}

This is a controlled before-and-after study [18] conducted from September to November 2019 in six rural areas of New Taipei City, Taiwan. Older adults were recruited from six public adult daycare centers. The participants of each adult daycare center were assigned in a counterbalanced order into the experimental and control groups. Each group comprised older adults from three adult daycare centers. The study protocol was approved by the Institutional Review Board of the Antai-Tian-Sheng Memorial Hospital, and the trial was registered in the ClinicalTrials.gov (NCT04311138).

The inclusion criteria were (1) aged 65 years or older; (2) had mild to moderate mobility deficits (de Morton Mobility Index (DEMMI) score 39-67 [19,20]); and (3) had a gait speed of $\leq 1 \mathrm{~m} / \mathrm{s}$ [21]. The exclusion criteria included the following: had moderate to severe cognitive impairment or a Clinical Dementia Rating score of $\geq 2$ [22]. All participants provided signed informed consent prior to participating.

\subsection{Intervention}

\subsubsection{PCHER}

The PCHER program is a community-based intervention program. Adult daycare centers for the experimental group were visited by two rehabilitation specialists (i.e., qualified physiotherapist or occupational therapist) weekly for intervention. Each intervention session lasted $2.5 \mathrm{~h}$, comprising $1.5 \mathrm{~h}$ of group courses and $1 \mathrm{~h}$ of individualized reablement training. The intervention spanned 10 weeks

The group training course covered physical training, cognitive training, and health education, which was designed according to problems frequently encountered by older adults in their daily lives. Training was led by a therapist using presentation slides. Both knowledge and skill were taught prior to practice (Table 1). After the group course ended, 
therapists provided one-on-one reablement training to each participant. At the initial visit assessment, a care goal was formulated after discussion between participants and therapists to identify the problems they encountered and perceived as the most important in performing self-care, productive activities, and leisure activities.

Table 1. Group-based physical-cognitive training and health education.

\begin{tabular}{|c|c|}
\hline Week & Content \\
\hline 1 st & $\begin{array}{l}\text { Sit-to-stand exercise (10 repetitions/set, } 10 \text { sets) } \\
\text { Cognicise [23] (e.g., marching with simultaneously clapping, subtracting or adding numbers) }\end{array}$ \\
\hline 2nd & $\begin{array}{l}\text { Static stretching exercises: Neck, shoulder, forearm, spine, gluteus, thigh and calf }(10 \mathrm{~min} / \mathrm{set}) \\
\text { Cognitive finger exercises with various gestures }\end{array}$ \\
\hline 3 rd & $\begin{array}{l}\text { Pelvic floor exercises ( } 5 \text { s contraction and } 20 \text { s resting, } 10 \text { repetitions / set) } \\
\text { Health education: Improving sleep quality }\end{array}$ \\
\hline 4 th & $\begin{array}{l}\text { Fall prevention and balance exercise: Squat, heel raises, and lunge (each } 10 \text { repetitions/set) } \\
\text { Health education: Antidementia diets }\end{array}$ \\
\hline 5 th & $\begin{array}{l}\text { Exercise: How to get back up after a fall ( } 3 \text { repetitions) } \\
\text { Health education: Long-term care resources and assistive device application }\end{array}$ \\
\hline 6th & $\begin{array}{l}\text { Squatting exercise (10 repetitions/set, } 10 \text { sets) } \\
\text { Health education: Oral exercise }\end{array}$ \\
\hline 7th & $\begin{array}{l}\text { Spinal decompression and stretching exercise }(20 \mathrm{~min}) \\
\text { Visual processing exercise (e.g., hands up) }\end{array}$ \\
\hline 8th & $\begin{array}{l}\text { Cognitive games and brain exercises: Visual attention, visual spatial and closure, and visual discrimination } \\
\text { Abdominal breathing exercise ( } 5 \text { times/minute, } 5 \text { repetitions } / \text { set) }\end{array}$ \\
\hline 9th & $\begin{array}{l}\text { Aerobic exercise: Walking }(20 \mathrm{~min}) \\
\text { Health education: Medication management }\end{array}$ \\
\hline 10th & Antidementia: The five senses \\
\hline
\end{tabular}

\subsubsection{Physical-Cognitive Training and Health Education (PCHE)}

The control group also received $1.5 \mathrm{~h}$ of group courses (Table 1), but, in place of individualized reablement intervention, they were given placebo treatments, including therapists to accompany and chat with them in sitting position.

\subsection{Outcomes}

The main outcome measurements included mobility, cognitive function, ADL function, physical function, and self-perceived activity performance and satisfaction with performance. Assessments were performed before and after the interventions by a blinded examiner.

Mobility function was measured using the DEMMI. The DEMMI is a 15-item unidimensional instrument that measures mobility across the spectrum from bed bound to independent mobility and has been rigorously developed and validated [19]. The Rasch converted interval DEMMI score ranges from 0 to 100, with higher scores indicating better mobility. A DEMMI score of 20-36, 39-67, and 74-100 signify severe, mild to moderate, and no mobility difficulty, respectively [20].

Cognitive function was assessed using the Saint Louis University Mental Status (SLUMS) Examination, which was validated using a sample of community-dwelling older adults [24]. The SLUMS is a 30-point questionnaire that tests for orientation, memory, attention, and executive functions. The mild cognitive impairment (MCI) cutoff scores for individuals with at least high school education and less than high school education were 21-26 and 20-24, respectively. The dementia cutoff scores for individuals with at least high school education and less than high school education were $<21$ and $<20$, respectively [25]. In this study, we used SLUMS-Chinese version [26]; the scores to detect dementia for Chinese population with at least high school education and less than high school education were $<24$ and $<22$, respectively [27]. 
The Barthel Index (BI) is a reliable and valid tool for measuring ADL function [28]. It contains the following 10 items: feeding, grooming, dressing, bathing, bowel control, bladder control, toileting, transferring, ambulation, and stairs climbing. The total score ranges from 0 to 100; a score of 0-20 indicates "total" dependency, 21-60 indicates "severe" dependency, 61-90 indicates "moderate" dependency, and 91-99 indicates "slight" dependency.

The Short Physical Performance Battery (SPPB), a valid tool, was applied to assess physical performance, including standing balance, walking, and five times sit-to-stand [8]. For each test, the time required was recorded and converted into points $(0-4)$, thereby giving a total score of $0-12$ points, with higher scores representing better physical function. A total score $\leq 9$ points can distinguish frail from non-frail individuals [29] and is used as low physical performance criteria for sarcopenia [30].

The Canadian Occupational Performance Measure (COPM), with adequate psychometric properties, was used to measure participants' self-perceived activity performance (COPM_P) and satisfaction with performance (COPM_S) within the following 3 areas: self-care, productivity, and leisure [31]. During a semi-structured interview, participants were asked to prioritize five of the most important activities and thereafter rate the level of performance and level of satisfaction on a scale from 1 to 10. A higher score reflects better performance and higher satisfaction.

\subsection{Statistical Analyses}

Statistical analysis was performed using SPSS 23.0 (IBM, Armonk, NY, USA). ShapiroWilk test was employed to test for data normality. The Chi-square test and Mann-Whitney $U$ test were conducted to determine differences between groups with respect to baseline characteristics of the participants and pre-intervention scores. The Wilcoxon signed-rank test was subsequently employed for comparison outcome variables of interest between preand post-assessments for each group, and the Mann-Whitney $U$ test was used to examine the differences in score changes between groups. The statistical significance was set at $\alpha=0.05$. Effect sizes for nonparametric data were calculated with the formula $r=Z / \sqrt{ } n$, and classified as small $(r=0.1)$, medium $(r=0.3)$, and large $(r=0.5)$ [32].

\section{Results}

\subsection{Participants}

Twenty-eight elders were enrolled in the study from six adult daycare centers. The PCHER group and the PCHE group enrolled 16 elders (six, six, and four from the three centers) and 12 elders (seven, two, and three from the three centers), respectively; the response rate ranges from 20 to $47 \%$. All of them completed the study. The demographic information for the study participants is presented in Table 2. The two groups did not exhibit significant differences except education attainment and illness. The participants in the PCHER group had higher education and a higher percentage of diabetes.

\subsection{Outcomes}

The pre-intervention scores were similar between the PCHER and PCHE groups except for the SPPB $(p=0.01)$, walking $(p=0.24)$, and five times sit-to-stand $(p=0.01)$ scores (Table 3). No statistically significant differences were found in the comparison of post-intervention scores between the two groups. However, the PCHER group exhibited significant post-intervention improvements in the DEMMI, SLUMS, BI, SPPB, and COPM_S scores (all $p<0.05$, Table 3 ). In terms of the COPM_P, the improvement was marginally significant $(p=0.055)$, with a medium effect size $(r=0.48)$. The PCHE group only demonstrated significant improvements for the DEMMI and COPM_S scores. The PCHER group also demonstrated greater improvements in the SPPB scores than the PCHE group did $(p=0.02)$. In terms of effect size, the PCHER group demonstrated greater improvements on the DEMMI, SLUMS, BI, SPPB, and COPM_P scores when compared with those for the PCHE group. 
Table 2. Demographic information and basic characteristics of the participants.

\begin{tabular}{|c|c|c|c|}
\hline Characteristics & PCHER $(n=16)$ & PCHE $(n=12)$ & $p$-Value \\
\hline Gender & & & 0.227 \\
\hline Male & $6(37.5)$ & $2(16.7)$ & \\
\hline Female & $10(62.5)$ & $10(83.3)$ & \\
\hline Age & 79 (65-99) & $82(69-91)$ & 0.318 \\
\hline Body height & $157.5(145-170)$ & $155.5(145-167)$ & 0.415 \\
\hline Body weight & $61.9(40-86)$ & $53.0(38-80)$ & 0.150 \\
\hline Education & & & $0.030 *$ \\
\hline Elementary school and below & $10(62.5)$ & $12(100)$ & \\
\hline Middle school & $3(18.8)$ & 0 & \\
\hline High school & $2(12.5)$ & 0 & \\
\hline College and above & $1(6.2)$ & 0 & \\
\hline Solitary & $2(12.5)$ & $1(8.3)$ & 0.724 \\
\hline Illness & $1(0-3)$ & $0.5(0-1)$ & 0.050 \\
\hline Cardiovascular diseases & $4(25.0)$ & 0 & 0.061 \\
\hline Hypertension & $9(56.3)$ & $6(50.0)$ & 0.743 \\
\hline Diabetes & $5(31.3)$ & 0 & $0.033 *$ \\
\hline
\end{tabular}

PCHER = physical-cognitive training, health education, and reablement, $\mathrm{PCHE}=$ physical-cognitive training and health education. Data were presented as median (range) or number of cases (percentages). ${ }^{*} p<0.05$.

Table 3. Comparisons of mobility, cognitive function, ADL function, physical function, and self-perceived activity performance and satisfaction with performance.

\begin{tabular}{|c|c|c|c|c|c|c|c|c|}
\hline \multirow[b]{2}{*}{ Outcome } & \multicolumn{4}{|c|}{ PCHER $(n=16)$} & \multicolumn{4}{|c|}{ PCHE $(n=12)$} \\
\hline & $\begin{array}{c}\text { Pre- } \\
\text { Intervention }\end{array}$ & $\begin{array}{c}\text { Post- } \\
\text { Intervention }\end{array}$ & $p$ & $\begin{array}{l}\text { Effect } \\
\text { Size }\end{array}$ & $\begin{array}{c}\text { Pre- } \\
\text { Intervention }\end{array}$ & $\begin{array}{c}\text { Post- } \\
\text { Intervention }\end{array}$ & $p$-Value & $\begin{array}{l}\text { Effect } \\
\text { Size }\end{array}$ \\
\hline DEMMI & $57(41-67)$ & $67(44-100)$ & $0.007 *$ & 0.68 & $67(53-67)$ & $67(48-100)$ & 0.046 * & 0.58 \\
\hline SLUMS & $19(3-27)$ & $21.5(3-30)$ & 0.028 * & 0.55 & $14(6-27)$ & $17(3-30)$ & 0.610 & 0.15 \\
\hline BI & $95(85-100)$ & $100(85-100)$ & 0.026 * & 0.56 & $97.5(90-100)$ & $100(85-100)$ & 0.414 & 0.24 \\
\hline SPPB & $6(2-9)$ & $8(3-12)$ & $0.005 *$ & 0.70 & $9(5-12)$ & $9(5-12)$ & 0.915 & 0.03 \\
\hline Balance & $3.5(0-4)$ & $4(1-4)$ & 0.161 & 0.35 & $4(2-4)$ & $4(2-4)$ & 0.317 & 0.29 \\
\hline Walking & $2(1-3)$ & $2(1-4)$ & $0.010 *$ & 0.65 & $2.5(1-4)$ & $2(1-4)$ & 1.000 & 0 \\
\hline $\begin{array}{l}\text { Five times sit-to-stand } \\
\text { COPM }\end{array}$ & $1(0-3)$ & $2(0-4)$ & 0.027 * & 0.55 & $3(1-4)$ & $2.5(1-4)$ & 0.593 & 0.15 \\
\hline Performance & $4.5(1-10)$ & $5(2-10)$ & 0.055 & 0.48 & $5(3-10)$ & $6(3-10)$ & 0.223 & 0.35 \\
\hline Satisfaction & $5(3-10)$ & $5.5(3-10)$ & 0.048 * & 0.49 & $5(1-10)$ & $6(3-10)$ & 0.017 * & 0.69 \\
\hline
\end{tabular}

DEMMI = de Morton Mobility Index, SLUMS = Saint Louis University Mental Status Examination, BI = Barthel index, SPPB = Short Physical Performance Battery, COPM = Canadian Occupational Performance Measure. Data were presented as median (range). ${ }^{*}$ Significant differences between pre- and post-intervention $(p<0.05)$.

\section{Discussion}

The present study found that combining individualized reablement and group-based physical-cognitive training and health education had greater effects on lower extremity function, mobility, cognitive function, and ADL function, relative to a control, among rural community-dwelling older adults with mild to moderate mobility deficits. Our findings added knowledge to reablement in a community-based context. As functional abilities are essential indicators of reablement [1,3,5], our findings support the potential use of reablement in the context of community for improving the mobility and functional independence of community-dwelling older adults.

This reablement study considered older adults with mild to moderate mobility deficits. The BI assessment (>90) indicated they were mildly dependent. However, the DEMMI and SPPB scores indicated that functional mobility decline or deficits influenced their physical performance. The evidence suggests that physical exercise interventions could improve the performance-based measures of physical function (e.g., gait speed and SPPB scores) in community-dwelling, frail older adults [9,33]. The combined center- and homebased multicomponent exercise programs also improved physical performance in the older 
adults [34]. Additionally, research on home-based reablement revealed an improvement of the SPPB after a period of reablement service [35,36]. The current study findings corroborate those of previous studies indicating a combination of group exercise and individualized reablement effectively improved mobility and physical function in older adults, thereby improving their everyday functionality. The positive result observed by this study was related to the multicomponent lower extremity training provided in the PCHER program. However, the significant changes of the SPPB, walking, and the five times sit-to-stand scores in the PCHER group should be interpreted with caution because the lower baseline scores of this group made the room for improvement larger.

The items represented in the SPPB were markers for identifying preclinical disability in community-dwelling older adults [37]. A change of 1.0 point for the SPPB and $0.10 \mathrm{~m} / \mathrm{s}$ for gait speed were considered substantial [38]. The score changes of the SPPB (1.9-point) in our study were higher than those of reablement studies $[35,36]$. After the PCHER, time taken for 4-m walking test significantly improved from $10.9 \pm 7.5$ to $7.7 \pm 3.8 \mathrm{~s}$ (gait speed: from $0.5 \pm 0.2$ to $0.6 \pm 0.2 \mathrm{~m} / \mathrm{s}$ ); the time taken to complete five sit-to-stand significantly improved from $24.5 \pm 14.1$ to $18.1 \pm 15.0 \mathrm{~s}$. Despite the significant improvement of the SPPB, nine (75\%) participants in the PCHER group had a post-intervention score $\leq 9$, suggesting a frail or sarcopenia risk [29,30]. Exercise twice a week and a contribution of 1.0, 1.2 to $1.5 \mathrm{~g} / \mathrm{kg}$ of protein/day and $3 \mathrm{~g}$ of beta-hydroxy-beta-methylbutyrate (HMB, leucine precursor) per day with a supplementation period of 8 to 12 weeks enhanced leg muscle mass and knee extensor strength [39]. Future studies could increase the exercise frequency or nutritional supplementation to improve the muscle mass, strength, and function in older adults.

The participants with mild to moderate mobility difficulty in the present study had a DEMMI score of 41-67. The finding was in line with those of a previous study that found that community-dwelling older adults who ambulated with an aid had a DEMMI score of $64.1 \pm 12.4$ [40]. A Taiwan study showed the elderly with a DEMMI score of 39-67 frequently had disabled ADL items of climbing and ambulation [20]. Additionally, DEMMI scores $<48$ and $<67$ could predict an inability to ambulate close to the participant's residence and to use public transportation, respectively [41]. Community ambulation are crucial for older adults to leave their homes and mingle with their community. After PCHER intervention, the mean DEMMI score exhibited a significant 15.3-point increase. The improvement is clinically meaningful and relevant because a score change of 13 points or larger could be considered significant and clinically important in the community setting [40].

In addition to the improvement of functional abilities using objective measures, the PCHER group also showed subjective improvements in the COPM_P and the COPM_S with medium-to-large effect sizes. Interestingly, the PCHE group also reported increased levels of the COPM_S. A similar phenomenon has been reported in previous studies [2,36]. The authors suggest that the improvement may be caused by the therapeutic effect of the baseline COPM interview, which increases the control group's awareness of their activity limitations and prompts them to seek solutions themselves [2]. Another possible explanation is the participants in the PCHE group received companionship from the therapists.

Cognitive training can be effective in improving various aspects of objective cognitive functioning, including memory performance, executive functioning, processing speed, attention, fluid intelligence, and subjective cognitive performance [42]. In the current study, the cognitive functions of older adults significantly improved in the PCHER group but not in the PCHE group, indicating that a 1.5-h group course, comprising a cognitive training component, could only maintain cognitive function. We could not eliminate the possibility that the fact that cognitive functions did not improve in the PCHE group may be due to the lower educational level of this group. The possible benefit of an addition of an individualized reablement component on cognition may be associated with physical activity or exercise, which are common strategies integrated in the reablement intervention [43]. The studies on older subjects with MCI reported some positive effects of 
physical exercise on cognition, mainly on global cognition, executive function, attention, and delayed recall [44].

Lower mean years of education $(<6)$ and poor cognitive function were reported for rural-dwelling older adults [12]. Most of our participants were illiterate $(35.7 \%)$ or had an elementary school level of education (32.1\%). We noticed that they had a low SLUMS score of 14-19. For individuals with an education level lower than high school, the SLUMS scores $<20$ [26] or 22 [27] indicate dementia. However, relevant studies have not focused on older adults with extremely low education levels. Nevertheless, because of impaired cognitive function, intervention targeting mental health is recommended for rural-dwelling older adults.

The present study had some limitations. First, the trial was not randomized, and the sample size was small, which limits the generalizability of our findings. A sample size of 12 per group met the rule of thumb for a pilot study [45]. Second, the intervention frequency was only once weekly because the therapists were required to travel to six rural adult daycare centers to perform the interventions. Third, only older adults with mild to moderate mobility deficits were recruited because individualized reablement training was provided by two therapists. The dilemmas, however, are faced while delivering reablement services to a remote area. Finally, we did not conduct follow-up measurements. Taken together, future studies may utilize a high-quality design, enroll more participants, and integrate more resources to increase the intervention frequency or duration to accumulate evidence-based information that can inform best practices.

\section{Conclusions}

This study verified that combining individualized reablement with group-based multicomponent training was superior to group courses alone in enhancing the functional abilities of community-dwelling older adults with mobility deficits. Future studies with follow-up measurements are warranted.

Author Contributions: Conceptualization, C.-Y.S. and P.-L.H.; data curation, P.-L.H.; funding acquisition, ADLers Occupational Therapy Clinic; investigation, P.-L.H. and ADLers Occupational Therapy Clinic; methodology, C.-Y.S. and P.-S.L.; writing_original draft, C.-Y.S.; writing—review \& editing, C.-Y.S. and P.-S.L. All authors have read and agreed to the published version of the manuscript.

Funding: This study was supported by a grant (Number: S108013) to the ADLers Occupational Therapy Clinic from the Department of Health, New Taipei City Government.

Institutional Review Board Statement: The study was conducted according to the guidelines of the Declaration of Helsinki, and approved by the Institutional Review Board of the Antai-Tian-Sheng Memorial Hospital (Approval Number: 19-060-B).

Informed Consent Statement: Informed consent was obtained from all subjects involved in the study.

Data Availability Statement: The datasets used and/or analyzed during the current study are available from the corresponding authors on reasonable request.

Acknowledgments: The authors thank the six public adult daycare centers for providing support. The authors would also like to thank all of the study participants.

Conflicts of Interest: The authors declare no conflict of interest.

\section{References}

1. Cochrane, A.; Furlong, M.; McGilloway, S.; Molloy, D.W.; Stevenson, M.; Donnelly, M. Time-limited home-care reablement services for maintaining and improving the functional independence of older adults. Cochrane Database Syst. Rev. 2016, 10, CD010825. [CrossRef]

2. Tuntland, H.; Aaslund, M.K.; Espehaug, B.; Forland, O.; Kjeken, I. Reablement in community-dwelling older adults: A randomised controlled trial. BMC Geriatr. 2015, 15, 145. [CrossRef]

3. Sims-Gould, J.; Tong, C.E.; Wallis-Mayer, L.; Ashe, M.C. Reablement, reactivation, rehabilitation and restorative interventions with older adults in receipt of home care: A systematic review. J. Am. Med. Dir. Assoc. 2017, 18, 653-663. [CrossRef] [PubMed] 
4. Lewin, G.; Concanen, K.; Youens, D. The Home Independence Program with non-health professionals as care managers: An evaluation. Clin. Interv. Aging 2016, 11, 807. [CrossRef]

5. Tessier, A.; Beaulieu, M.-D.; Mcginn, C.A.; Latulippe, R. Effectiveness of reablement: A systematic review. Healthc. Policy 2016, 11, 49. [CrossRef]

6. Hattori, S.; Yoshida, T.; Okumura, Y.; Kondo, K. Effects of reablement on the independence of community-dwelling older adults with mild disability: A randomized controlled trial. Int. J. Environ. Res. Public Health 2019, 16, 3954. [CrossRef]

7. Arnau, A.; Espaulella, J.; Serrarols, M.; Canudas, J.; Formiga, F.; Ferrer, M. Risk factors for functional decline in a population aged 75 years and older without total dependence: A one-year follow-up. Arch. Gerontol. Geriatr. 2016, 65, 239-247. [CrossRef]

8. Guralnik, J.M.; Ferrucci, L.; Simonsick, E.M.; Salive, M.E.; Wallace, R.B. Lower-extremity function in persons over the age of 70 years as a predictor of subsequent disability. N. Engl. J. Med. 1995, 332, 556-561. [CrossRef] [PubMed]

9. de Labra, C.; Guimaraes-Pinheiro, C.; Maseda, A.; Lorenzo, T.; Millan-Calenti, J.C. Effects of physical exercise interventions in frail older adults: A systematic review of randomized controlled trials. BMC Geriatr. 2015, 15, 154. [CrossRef] [PubMed]

10. Liao, Y.Y.; Chen, I.H.; Wang, R.Y. Effects of Kinect-based exergaming on frailty status and physical performance in prefrail and frail elderly: A randomized controlled trial. Sci. Rep. 2019, 9, 9353. [CrossRef]

11. Sanders, L.M.J.; Hortobágyi, T.; Karssemeijer, E.G.A.; Van der Zee, E.A.E.; Scherder, J.A.; van Heuvelen, M.J.G. Effects of low- and high-intensity physical exercise on physical and cognitive function in older persons with dementia: A randomized controlled trial. Alzheimers Res. Ther. 2020, 12, 28. [CrossRef] [PubMed]

12. Chuang, Y.F.; Liu, Y.C.; Tseng, H.Y.; Lin, P.X.; Li, C.Y.; Shih, M.H.; Lin, K.C.; Yang, T.O.; Yan, S.H.; Chiu, Y.L. Urban-rural differences in the prevalence and correlates of mild cognitive impairment in community-dwelling older adults in Taiwan: The EMCIT study. J. Formos. Med. Assoc. 2021, 120, 1749-1757. [CrossRef] [PubMed]

13. Lin, Y.J.; Wu, H.C.; Buo, B.M.; Chang, C.S.; Chang, Y.F.; Liu, P.Y.; Hou, M.T.; Huang, P.C.; Chen, C.Y.; Wu, C.H. Nutritional status and associated factors of the elderly in a remote community. Taiwan Geriatr. Gerontol. 2013, 8, 186-201.

14. Huang, L.H.; Chang, C.S.; Chang, Y.F.; Liu, P.Y.; Chiu, C.J.; Hou, M.T.; Huang, P.C.; Chen, C.Y.; Wu, H.C.; Hsu, Y.H.; et al. Epidemiological survey of falls and associated risk factors in the elderly living in a remote community. Taiwan Geriatr. Gerontol. 2013, 8, 273-289.

15. Wu, C.H.; Chen, K.T.; Hou, M.T.; Chang, Y.F.; Chang, C.S.; Liu, P.Y.; Wu, S.J.; Chiu, C.J.; Jou, I.M.; Chen, C.Y. Prevalence and associated factors of sarcopenia and severe sarcopenia in older Taiwanese living in rural community: The Tianliao Old People study 04. Geriatr. Gerontol. Int. 2014, 14 (Suppl. 1), 69-75. [CrossRef]

16. Lauenroth, A.; Ioannidis, A.E.; Teichmann, B. Influence of combined physical and cognitive training on cognition: A systematic review. BMC Geriatr. 2016, 16, 141. [CrossRef]

17. Cho, Y.H.; Mohamed, O.; White, B.; Singh-Carlson, S.; Krishnan, V. The effects of a multicomponent intervention program on clinical outcomes associated with falls in healthy older adults. Aging Clin. Exp. Res. 2018, 30, 1101-1110. [CrossRef]

18. Schmidt, W.P. Randomised and non-randomised studies to estimate the effect of community-level public health interventions: Definitions and methodological considerations. Emerg. Themes Epidemiol. 2017, 14, 9. [CrossRef]

19. de Morton, N.A.; Davidson, M.; Keating, J.L. The de Morton Mobility Index (DEMMI): An essential health index for an ageing world. Health Qual. Life Outcomes 2008, 6, 63. [CrossRef]

20. Yang, Y.A.; Tsauo, J.Y.; Lee, H.C.; Tsai, Y.J.; Lin, C.F.; Wang, C.Y. Activities of daily living disability and associated physical performances in older adults with mild to moderate mobility difficulty. Formos. J. Phys. Ther. 2018, 43, $203-214$.

21. Kyrdalen, I.L.; Thingstad, P.; Sandvik, L.; Ormstad, H. Associations between gait speed and well-known fall risk factors among community-dwelling older adults. Physiother. Res. Int. 2019, 24, e1743. [CrossRef]

22. Hughes, C.P.; Berg, L.; Danziger, W.L.; Coben, L.A.; Martin, R.L. A new clinical scale for the staging of dementia. Br. J. Psychiatry 1982, 140, 566-572. [CrossRef] [PubMed]

23. Suzuki, T.; Makizako, H.; Park, H.; Lee, S.; Tsutsumimoto, K.; Umemura, K.; Maki, Y.; Shimada, H. Community-based intervention for prevention of dementia in Japan. J. Prev. Alzheimers Dis. 2015, 2, 71-76.

24. Feliciano, L.; Horning, S.M.; Klebe, K.J.; Anderson, S.L.; Cornwell, E.; Harris, H.P. Utility of the SLUMS as a cognitive screening tool among a nonveteran sample of older adults. Am. J. Geriatr. Psychiatry 2013, 21, 623-630. [CrossRef] [PubMed]

25. Tariq, S.H.; Tumosa, N.; Chibnall, J.T.; Perry, M.H.; Morley, J.E. Comparison of the Saint Louis University mental status examination and the mini-mental state examination for detecting dementia and mild neurocognitive disorder: A pilot study. Am J. Geriatr. Psychiatry 2006, 14, 900-910. [CrossRef] [PubMed]

26. $\mathrm{Hu}, \mathrm{M} . \mathrm{H}$. New choice of a screening tool for cognitive function: The SLUMS test. J. Long-Term Care 2010, 14, 267-276.

27. Yang, Y.P.; Huang, Y.C.; Chen, C.S.; Yang, Y.C.; Wang, J.J. Sensitivity and specificity of the Saint Louis University Mental Status Examination to detect mild cognitive impairment and dementia in Chinese population. Gerontology 2021, 67, 152-159. [CrossRef] [PubMed]

28. Hsueh, I.P.; Lee, M.M.; Hsieh, C.L. Psychometric characteristics of the Barthel activities of daily living index in stroke patients. J. Formos. Med. Assoc. 2001, 100, 526-532.

29. da Camara, S.M.; Alvarado, B.E.; Guralnik, J.M.; Guerra, R.O.; Maciel, A.C. Using the short physical performance battery to screen for frailty in young-old adults with distinct socioeconomic conditions. Geriatr. Gerontol. Int. 2013, 13, 421-428. [CrossRef] 
30. Chen, L.K.; Woo, J.; Assantachai, P.; Auyeung, T.W.; Chou, M.Y.; Iijima, K.; Jang, H.C.; Kang, L.; Kim, M.; Kim, S.; et al. Asian Working Group for Sarcopenia: 2019 Consensus Update on Sarcopenia Diagnosis and Treatment. J. Am. Med. Dir. Assoc. 2020, 21, 300-307.e2. [CrossRef]

31. Tuntland, H.; Aaslund, M.K.; Langeland, E.; Espehaug, B.; Kjeken, I. Psychometric properties of the Canadian occupational performance measure in home-dwelling older adults. J. Multidiscip. Healthc. 2016, 6, 411-423. [CrossRef] [PubMed]

32. Fritz, C.O.; Morris, P.E.; Richler, J.J. Effect size estimates: Current use, calculations, and interpretation. J. Exp. Psychol. Gen. 2012, 141, 2-18. [CrossRef]

33. Giné-Garriga, M.; Roqué-Fíguls, M.; Coll-Planas, L.; Sitjà-Rabert, M.; Salvà, A. Physical exercise interventions for improving performance-based measures of physical function in community-dwelling, frail older adults: A systematic review and metaanalysis. Arch. Phys. Med. Rehabil. 2014, 95, 753-769.e3. [CrossRef] [PubMed]

34. Sadjapong, U.; Yodkeeree, S.; Sungkarat, S.; Siviroj, P. Multicomponent exercise program reduces frailty and inflammatory biomarkers and improves physical performance in community-dwelling older adults: A randomized controlled trial. Int. J. Environ. Res. Public Health 2020, 17, 3760. [CrossRef] [PubMed]

35. Parsons, J.G.; Sheridan, N.; Rouse, P.; Robinson, E.; Connolly, M. A randomized controlled trial to determine the effect of a model of restorative home care on physical function and social support among older people. Arch. Phys. Med. Rehabil. 2013, 94, 1015-1022. [CrossRef]

36. Langeland, E.; Tuntland, H.; Folkestad, B.; Førland, O.; Jacobsen, F.F.; Kjeken, I. A multicenter investigation of reablement in Norway: A clinical controlled trial. BMC Geriatr. 2019, 19, 29. [CrossRef]

37. Cavanaugh, E.J.; Richardson, J.; McCallum, C.A.; Wilhelm, M. The predictive validity of physical performance measures in determining markers of preclinical disability in community dwelling middle-aged and older adults: A systematic review. Phys. Ther. 2018, 98, 1010-1021. [CrossRef]

38. Perera, S.; Mody, S.H.; Woodman, R.C.; Studenski, S.A. Meaningful change and responsiveness in common physical performance measures in older adults. J. Am. Geriatr. Soc. 2006, 54, 743-749. [CrossRef]

39. Kim, H.K.; Suzuki, T.; Saito, K.; Yoshida, H.; Kobayashi, H.; Kato, H.; Katayama, M. Effects of exercise and amino acid supplementation on body composition and physical function in community-dwelling elderly Japanese sarcopenic women: A randomized controlled trial. J. Am. Geriatr. Soc. 2012, 60, 16-23. [CrossRef]

40. Davenport, S.J.; de Morton, N.A. Clinimetric properties of the de Morton Mobility Index in healthy, community-dwelling older adults. Arch. Phys. Med. Rehabil. 2011, 92, 51-58. [CrossRef]

41. Søndergaard, K.; Petersen, L.E.; Pedersen, M.K.; Svendsen, A.B.; Juhl, C.B. Disabil. The responsiveness and predictive validity of the de Morton Mobility Index in geriatric rehabilitation. Disabil. Rehabil. 2020. [CrossRef] [PubMed]

42. Reijnders, J.; van Heugten, C.; van Boxtel, M. Cognitive interventions in healthy older adults and people with mild cognitive impairment: A systematic review. Ageing Res. Rev. 2013, 12, 263-275. [CrossRef]

43. Mjøsund, H.L.; Moe, C.F.; Burton, E.; Uhrenfeldt, L. Integration of physical activity in reablement for community dwelling older adults: A systematic scoping review. J. Multidiscip. Healthc. 2020, 13, 1291-1315. [CrossRef] [PubMed]

44. Öhman, H.; Savikko, N.; Strandberg, T.E.; Pitkälä, K.H. Effect of physical exercise on cognitive performance in older adults with mild cognitive impairment or dementia: A systematic review. Dement. Geriatr. Cogn. Disord. 2014, 38, 347-365. [CrossRef] [PubMed]

45. Julious, S.A. Sample size of 12 per group rule of thumb for a pilot study. Pharm. Stat. 2005, 4, 287-291. [CrossRef] 